\title{
Is There Any Rationale for Detecting Hormone Receptor/HER2 Status with Rebiopsy Without Progression Upfront Metastatic Breast Cancer? with 2 Cases
}

\author{
Berna Bozkurt Duman*, and Timuçin Çil \\ Department of Medical Oncology, University of Health Sciences, Adana City Education and Research Hospital, Adana, Turkey
}

${ }^{*}$ Corresponding author: Berna Bozkurt Duman, Department of Medical Oncology, University of Health Sciences, Adana City Education and Research Hospital, Adana, 01230, Turkey, Phone: +903224559000; E-mail: berboz@hotmail.com

Received: 23 Jan, 2021 | Accepted: 15 Feb, 2021 | Published: 26 Feb, 2021

Citation: Duman BB, Çil T (2021) Is There Any Rationale for Detecting Hormone Receptor/HER2 Status with Rebiopsy Without Progression Upfront Metastatic Breast Cancer? with 2 Cases. Int J Cancer Res Mol Mech 6(1): dx.doi.org/10.16966/2381-3318.148

Copyright: (C) 2021 Duman BB, et al. This is an open-access article distributed under the terms of the Creative Commons Attribution License, which permits unrestricted use, distribution, and reproduction in any medium, provided the original author and source are credited.

\begin{abstract}
Alteration of biomarkers is well-documented in breast cancer at locoregional recurrence or metastasis attributed to tumor heterogeneity and change in biology. There is some data about discordance between primary and metastatic sites. At the same time hormone, receptor status can change after neoadjuvant treatment and at the time of recurrence. Metastatic breast cancer without progression or recurrence after the targeted chemotherapy combination for planning maintenance therapy in Human epidermal growth factor receptor 2 (HER2) overexpression positive hormone receptors positive or triple-negative patient after chemotherapy. In guidelines, the time of rebiopsy has no exact time, if the time of biopsy is usually after the progression of the tumor. We presented cases in which we detected different hormone receptor statuses from the beginning without progression and before deciding on maintenance therapy. This subject is important for deciding therapy in the aspect of heterogeneous tumors like breast cancer. The important decision of rebiopsy time is debate. In this aspect, these two cases are important examples for these kinds of patients tumor heterogeneity in breast cancer is one of the most widely known entities. We found that two patients, one of whom was estrogen progesterone receptor negative HER2 $3(+++)$ at the time of diagnosis and the other who was triple negative at the time of diagnosis, had positive hormone receptors in the re-biopsies without progression. We aimed to discuss the tumor heterogeneity and timing of rebiopsy in breast cancer in the light of two cases.
\end{abstract}

Keywords: Breast cancer; Rebiopsy; Estrogen receptor; Progesterone receptor; HER2/neu

\section{Background}

Hormone receptor status, HER2 over expression, is significant factors in determining appropriate treatment and has both prognostic and predictive importance. These receptors status changes over time and breast cancer have heterogeneous behavior. There is no exact data for rebiopsy time in the recurrent patient [1]. A biopsy of first recurrence or metastatic disease is recommended to re-evaluate estrogen receptor status in patients with breast cancer and to select an appropriate treatment. Rebiopsy should be performed without recurrence and/or progression. Alteration of biomarkers is welldocumented in breast cancer at locoregional recurrence or metastasis attributed to tumor heterogeneity and change in biology. There is a lack of literature on alteration of biomarkers in metastatic breast cancer (MBC) at progression. There is no data about reassessment without progression of the disease.

\section{Case Report}

\section{Case 1}

45 years old premenopausal woman admitted to our department under the guidance of a Surgeon after a biopsy. In Positron Emission Tomography (PET) scan the primary tumor in the left breast and there are several metastases in the left supraclavicular, left axillary, left interpectoral hypermetabolic lymph nodes, multiple metastatic nodules in the liver, there are several lytic bone metastasis (right spin scapula, sternum, left the first costa, thoracic 8,10 vertebra and lumbar 2 vertebra, left iliac and right acetabulum). The tumor was invasive ductal breast carcinoma Estrogen Receptor (ER) negative, Progesterone receptor (PR) negative, Her2 3(+). Pertuzumab, Trastuzumab, Docetaxel combination therapy was given to the patient. After 3 cycles response was detected by PET scan. There was a near-complete response. Grade 3,4 toxicity was not seen during the therapy. After 6 cycles Docetaxel was removed from the combination due to toxicity. Maintenance therapy was given with pertuzumab and trastuzumab ECHO was normal during the therapy. No other finding was found in pet bt other than only a few bone lesions. Rebiopsy was performed from 2 vertebras. The pathology report was breast cancer metastasis ER positive in the percentage of $50 \%$, $P R$ was positive in the percentage of $1 \%$, HER2 was $3(+)$. Paraffin blocks were removed from the archive and re-evaluated by 2 different pathologists. The first and subsequent biopsies were re-examined. It was noted that hormone receptors, as stated in the first pathologies, were negative but later became positive. Tamoxifen was added to the combination therapy. The patient has been followed for approximately 21 months without any progression. Without disease progression headache started in the patient 21 months after the diagnosis. Cranial 
MRI was performed. Brain metastasis was detected. Cranial radiation therapy was begun.

\section{Case 2}

64 years old postmenopausal woman admitted to our department with pathology confirmed breast carcinoma. The subtype of breast cancer was mucinous carcinoma and triple negative morphology on immunohistochemical examination. In the PET scan; the primary tumor was in the left breast, numerous pathological lymph nodes were detected in the mediastinal and abdominal lymph node stations and the focal lesion in the descending colon. Endoscopy and colonoscopy were performed. We didn't find any pathologic findings in the location that was described in the PET scan in the descending colon. At the time of diagnosis, the patient who was diagnosed with metastatic triple negative breast cancer was given 4 cycles of anthracycline cyclophosphamide combination first, and after 4 cycles, tumor markers increased and disease progression was detected on PET-CT.

Single agent weekly paclitaxel in the dosage of $80 \mathrm{mg} / \mathrm{m}^{2}$ treatment was applied in the second line because the patient had many comorbidities such as diabetes and hypertension and she did not tolerated combination therapy very well. However, at the $7^{\text {th }}$ treatment the patient did not want to continue systemic chemotherapy due to toxicity. Already, the pdll expression of the patient was found to be negative and the BRCA1 and BRCA2 gene mutations were also negative There was a partial response in PET-scan after 7 weeks of paclitaxel treatment. Biopsy was taken from the left breast again. ER was found positive in the percentage of 35-40 PR was found positive in the percentage of 35-40 Her2 was found negative. Paraffin blocks were removed from the archive and re-evaluated by 2 different pathologists. The first and subsequent biopsies were re-examined. It was noted that hormone receptors, as stated in the first pathologies, were negative but later became positive. Aromatase inhibitör was given to the patient. The patient has been followed for approximately 21 months without any progression.

\section{Discussion and Conclusion}

HER2 overexpression, hormone receptor expression (estrogen receptor(ER) and progesterone receptor(PR)) are important factors for deciding treatment choices and prognosis in breast cancer.

Rebiopsy in breast cancer is very important for assessing particularly ER, PR, and HER2 overexpression. This is especially important if the primary cancer was deemed negative for ER, PR, and/ or HER2. Changes in receptor status affect the treatment decision. The discordance between primary tumor site and metastatic side can be seen in some patient [1-4].

For two hundred eighty-nine patients from two prospective studies, the discordance rate for ER, PR, and HER2 between the primary and recurrent disease was 13,31 , and $5.5 \%$, respectively [3]. The results of the biopsy were changed in 14 percent of patients due to re-biopsy.

One hundred seventy-eight patients from prospective observational women, the conversion rate between primary and metastatic disease similar to the findings like the other studies, with a discordance rate of 13, 28, and 3 percent for ER, PR, and HER2, respectively [4]. Among those patients who demonstrated a conversion in receptor status, ER $(\mathrm{n}=22)$ and $\mathrm{PR}(\mathrm{n}=47)$ status converted from positive to negative (78 and $72 \%$, respectively), and for HER2 status $(n=5)$, all went from positive to negative. Several studies have shown the changes of ER, PR, HER2 at the time of metastasis in early and locally advanced breast cancer [1-5].
There are several series that shows the difference in receptor status in recurrence or after neoadjuvant therapy. In one series with 32 patients of upfront metastatic breast cancer. In this study, changes were detected after progression in upfront metastatic breast cancer [5].

National comprehensive clinical network and American Society of Clinical Oncology/College of American Pathologists guideline recommend repeating receptor status in primary and metastatic breast cancer (MBC). However, there are no guidelines for repetition of receptor status in $\mathrm{MBC}$ patients at progression. Receptor alteration signifies that disease progression is associated with a change in tumor biology or tumor heterogeneity.

A biopsy (preferably providing histology) of a metastatic lesion should be performed, if easily accessible, to confirm diagnosis, particularly when metastasis is diagnosed for the first time. Due to ESMO ABC5 suggestions. Biological markers (especially HR and HER2) should be reassessed at least once in the metastatic setting, if clinically feasible. If the results of tumor biology in the metastatic lesion differ from the primary tumor, it is currently unknown which result should be used for treatment decision making. Since a clinical trial addressing this issue is difficult to undertake, the guidelines considering the use of targeted therapy (ET and/or anti-HER2 therapy) when receptors are positive in at least one biopsy, regardless of timing [6].

In metastatic breast cancer, due to tumor heterogeneity, it is generally recommended to take biopsy from the first recurrence or, if possible, from the metastatic area. However, taking a biopsy may not always be possible due to technical conditions. In a related prospective study, positive data were determined that $16 \alpha-\left[{ }^{18} \mathrm{~F}\right]$ fluoro$17 \beta$-oestradiol $\left({ }^{18} \mathrm{~F}\right.$-FES) PET-CT examination can give an idea about hormone receptor positivity. But still the gold standard is tissue reconstruction [7].

It is clear that breast cancer is a heterogeneous tumor. Two types of tumor heterogeneity are defined: intertumor heterogeneity and intra-tumor heterogeneity. Analyzes on the heterogeneity of breast cancer have resulted in a molecular classification of breast cancers that distinguishes four subtypes: Luminal A, Luminal B, HER 2+, and basal-like. However, with the primary intra-tumor heterogeneity of the tumor, it was revealed that there was discordance between the primary tumor and its metastasis, where hormone receptors and HER2 changed over time [8].

It is a well-known concept that breast cancer is a heterogeneous tumor. Rebiopsy is an approach recommended by the guidelines in locoregional or metastatic recurrent disease. In upfront metastatic disease, rebiopsy is a non-standard approach that has been investigated in a limited number of publications. Although the contribution of repeated biopsies to survival has not been clearly demonstrated, it contributes to offering new treatment options, but randomized studies are needed to illuminate this issue. In our case, while deciding on maintenance treatment, rebiopsy without progression guided the continuation of treatment in upfront metastatic disease.

In our cases, we documented changes in receptor status from the beginning without progression for deciding the maintenance therapy. Documentation of change in receptor status may be justified to determine maintenance therapy and prognosis in $\mathrm{MBC}$ without progression. The devil is in the details. 


\section{References}

1. Simmons C, Miller N, Geddie W, Gianfelice D, Oldfield M, et al. (2009) Does confirmatory tumor biopsy alter the management of breast cancer patients with distant metastases? Ann Oncol 20: 1499-1504.

2. Amir E, Clemons M, Purdie CA, Miller N, Quinlan P, et al. (2012) Tissue confirmation of disease recurrence in breast cancer patients: pooled analysis of multi-center, multi-disciplinary prospective studies. Cancer Treat Rev 38: 708-714.

3. de Dueñas EM, Hernández AL, Zotano AG, Carrión RMP, López-Muñiz JIC, et al. (2014) Prospective evaluation of the conversion rate in the receptor status between primary breast cancer and metastasis: results from the GEICAM 2009-03 ConvertHER study. Breast Cancer Res Treat 143: 507-515.

4. Swenerton KD, Legha SS, Smith T, Hortobagyi GN, Gehan EA, et al. (1979) Prognostic factors in metastatic breast cancer treated with combination chemotherapy. Cancer Res 39: 1552-1562.
5. Sharma M, Gogia A, Deo SSV, Mathur S (2018) Role of rebiopsy in metastatic breast cancer at progression. Curr Probl Cancer 43: 438 442.

6. Cardoso F, Paluch-Shimon S, Senkus E, Curigliano G, Aapro MS, et al. (2020) $5^{\text {th }}$ ESO-ESMO international consensus guidelines for advanced breast cancer (ABC 5). Ann Oncol 31: 1623-1649.

7. Chae SY, Ahn SH, Kim SB, Han S, Lee SH, et al. (2019) Diagnostic accuracy and safety of $16 \alpha-\left[{ }^{18} \mathrm{~F}\right]$ fluoro-17 $\beta$-oestradiol PET-CT for the assessment of oestrogen receptor status in recurrent or metastatic lesions in patients with breast cancer: a prospective cohort study. Lancet Oncol 20: 546-555.

8. Roulot $A$, Héquet $D$, Guinebretière $J M$, Vincent-Salomon $A$, Lerebours F, et al. (2016) Tumoral heterogeneity of breast cancer. Ann Biol Clin (Paris) 74: 653-660. 